\title{
The Role of Occlusion of the External Ear Canal in Hearing Loss
}

\author{
Original Investigation Dakshaini A. Narayanan (1), Rajagopalan Raman (1), Aun Wee Chong (1) \\ Department of Otorhinolaryngology, University Malaya, Kuala Lumpur, Malaysia
}

Abstract

ORCID IDs of the authors: D.A.N. 0000-0001-5237-4977; R.R. 0000-0003-4882-9398; A.W.C. 0000-0001-6928-7095.

Cite this article as: Narayanan DA, Raman $R$, Chong AW. The Role of Occlusion of the External Ear Canal in Hearing Loss. Turk Arch Otorhinolaryngol 2019; 57(3): 122-6.

\section{Corresponding Author:}

Dakshaini A.Narayanan; dakshaini83@gmail.com

Received Date: 25.11 .2018

Accepted Date: 22.07.2019

Content of this journal is licensed under a Creative Commons Attribution 4.0 International License. Available online at www.turkarchotolaryngol.net
Objective: The objectives are to determine and compare the degrees of hearing loss upon occlusion of the cartilaginous and the bony portion of the external ear canal (EAC).

Methods: There were 20 healthy participants with normal ears, and all gave an informed consent. After an otoscopy, a baseline pure tone audiogram (PTA) was conducted. If the PTA of the participant was normal, aqueous cream was applied with a syringe via an $18 \mathrm{G}$ cannula, from the tympanic membrane up to the isthmus which corresponds to the bony ear canal. A second PTA was conducted, and subsequently the cream was removed via suction under microscope guidance. The procedure was then repeated with the cream applied from the isthmus to the aperture of the external ear canal using the same cannula followed by a PTA and removal of cream under microscope.
Results: The mean threshold difference of occlusion at both portions of the ear canal were compared and analyzed. The mean threshold difference of hearing loss upon occlusion at the cartilaginous EAC was 37.5 to $48 \mathrm{~dB}$. The mean threshold difference of hearing loss upon occlusion at the bony EAC was less, with a range of 21 to $24.95 \mathrm{~dB}$. There was a statistical difference $(p<0.05)$ in the hearing loss between the blockage of the cartilaginous canal versus the blockage of the bony canal with a maximum difference at $2 \mathrm{kHz}$.

Conclusion: Cartilaginous block of the external ear canal causes more hearing loss than block of the bony ear canal. This correlates with the concept and properties of sound waves, resonance and impedance.

Keywords: External ear canal, conductive hearing loss, pure tone audiometry, comparison

\section{Introduction}

Human beings can hear sounds in the dynamic amplitude sensitivity range of almost 0 to 120 $\mathrm{dB}$ and in the frequency sensitivity bandwidth of approximately $0.02 \mathrm{kHz}$ to $20 \mathrm{kHz}$ (1). The adult external auditory canal (EAC) is divided into an outer one-third cartilaginous portion and an inner two-thirds bony portion. The overall length is approximately $2.5 \mathrm{~cm}$ (2). The isthmus is a bony-cartilaginous junction and corresponds to the narrowest point of the external auditory canal, which is situated at the junction of the outer one-third of the inner two-thirds of the canal (2). Besides sound transmission, the external ear canal performs a critical modification. Due to its shape and dimension, sounds in the $3000 \mathrm{~Hz}$ region resonate and are amplified (3). The overall modification effect of the outer ear is a $10-15 \mathrm{~dB}$ amplification of sound in the $2 \mathrm{kHz}$ to $4 \mathrm{kHz}$ range (3).

The objective of this study was to determine and compare the degrees of hearing loss upon an occlusion of the bony and the cartilaginous portions of the ear canal. The null hypothesis is that there is no difference in the degree of hearing loss upon occlusion of the bony or the cartilaginous portions. This study was done as an additional basic science knowledge of the application of physics of sounds in relation to the ear canal. The implications from this study may be related to conditions like keratosis obturans and canal cholesteatoma which cause milder hearing loss compared to the occlusion of the cartilaginous portion with ear wax. 


\section{Method}

The study included 20 participants, and all participants signed a written informed consent. All participants were female. Approval for the study was obtained from the Ethics Committee for Clinical Research of University Hospital (Approval date: 4.1.17, Approval number: MECID No 2016103-4317). The inclusion criteria were adults aged between 18-40 years with normal hearing. The exclusion criteria were any external or middle ear pathologies, pre-existing sensorineural hearing loss, vertigo, psychiatric condition and presence of known allergy to aqueous cream.

The participants underwent an otoscopy examination. A baseline pure tone audiogram (PTA) ranging from $0.25 \mathrm{kHz}$ up to $4 \mathrm{kHz}$ was conducted using the Madsen ITERA (Otometrics Natus, Denmark) clinical audiometer. If the PTA of the participant was normal, then an examination under microscope (EUM) was done. After EUM, aqueous cream was applied with a syringe via an $18 \mathrm{G}$ cannula with the needle removed. The cream was applied from the tympanic membrane up to the isthmus which corresponds to the bony ear canal and confirmed by pulling the pinna with the guidance of a microscope. The isthmus was used as a landmark to demarcate the boundary between the bony and the cartilaginous ear canals. Upon application of the cream, a second PTA was conducted and subsequently the cream was removed via suction under microscope guidance. The procedure was then repeated with the cream applied from the isthmus to the aperture of the external ear canal using the same cannula followed by a PTA and removal of cream under a microscope. In both cases PTA was done immediately after the cream was applied and the cream stayed in situ throughout the audiological assessment. The bony ear canal was not affected by the application of the cream at the cartilaginous portion since the isthmus was used as a clinical landmark and applied under microscopic guidance.

Aqueous cream was chosen to be applied at the external ear canal. Aqueous cream is a light, hydrocarbon-based emulsion. It is used as a topical, external medicine and emollient moisturizer. Aqueous cream contains emulsifying wax of $8 \% \mathrm{w} / \mathrm{w}$, white soft paraffin $12 \% \mathrm{w} / \mathrm{w}$ and liquid paraffin $8 \% \mathrm{w} / \mathrm{w}$ with preservatives comprising methylparaben $0.32 \% \mathrm{w} / \mathrm{w}$ and propylparaben $0.048 \% \mathrm{w} / \mathrm{w}$. The cream was manufactured by UPHA Pharmaceutical company which is a Malaysian based company. None of the participants developed any allergic reaction following the application of the aqueous cream. Aqueous cream was the choice for this research because it is easily available and cost effective.

\section{Statistical analysis}

Statistical analysis was performed using the Statistical Package for the Social Sciences version 24 (IBM Corp.; Armonk, NY, USA). Mean threshold difference, standard deviation and paired $\mathrm{T}$ test were performed.

\section{Results}

A total of 20 subjects took part in this study. Majority of the subjects were in their mid-twenties; their mean age was $24 \pm 2.72$ and all were female.
Table 1 provides the mean thresholds over different frequencies upon occlusion of the cartilaginous EAC. In general, the mean threshold differences across frequencies varied by as much as 48 $\mathrm{dB}$ to as little as $37.5 \mathrm{~dB}$. The highest level of hearing loss was recorded at $2 \mathrm{kHz}$ with a mean of $48 \mathrm{~dB} \pm 7.5$.

Table 2 illustrates the mean thresholds over different frequencies upon occlusion of the bony EAC. The overall mean threshold differences increased as the frequency was increased, with the exception at $4 \mathrm{kHz}$, which showed similar results to the occlusion of the cartilaginous EAC. The highest level of hearing loss was recorded at $2 \mathrm{kHz}$ with a mean of $24.95 \mathrm{~dB} \pm 6.77$. The standard error is a small value with a narrow margin which shows that the variability of the sample is small.

Figure 1 shows a comparison of the mean hearing losses ( $\mathrm{dB}$ HL) of occlusion at the bone and the cartilaginous EAC. As

Table 1. Mean hearing losses at different frequencies for occlusion of cartilaginous EAC

\begin{tabular}{|l|c|c|c|}
\hline Frequency $(\mathrm{kHz})$ & Mean $(\mathrm{dB})$ & $\mathrm{SD}$ & $\mathrm{SE}$ \\
\hline 0.25 & 37.50 & 8.19 & 1.83 \\
\hline 0.50 & 40.25 & 5.25 & 1.17 \\
\hline 1 & 46.00 & 6.61 & 1.48 \\
\hline 2 & 48.00 & 7.50 & 1.68 \\
\hline 4 & 42.25 & 7.69 & 1.72 \\
\hline
\end{tabular}

SD: standard deviation; SE: standard error

Table 2. Mean hearing losses at different frequencies for occlusion of bony EAC

\begin{tabular}{|l|c|c|c}
\hline Frequency $(\mathrm{Hz})$ & Mean $(\mathrm{dB})$ & SD & SE \\
\hline 0.25 & 21.75 & 5.20 & 1.16 \\
\hline 0.50 & 23.25 & 3.35 & 0.75 \\
\hline 1 & 24.75 & 4.13 & 0.92 \\
\hline 2 & 24.95 & 6.77 & 1.51 \\
\hline 4 & 21.50 & 10.00 & 2.24 \\
\hline
\end{tabular}

SD: standard deviation; SE: standard error

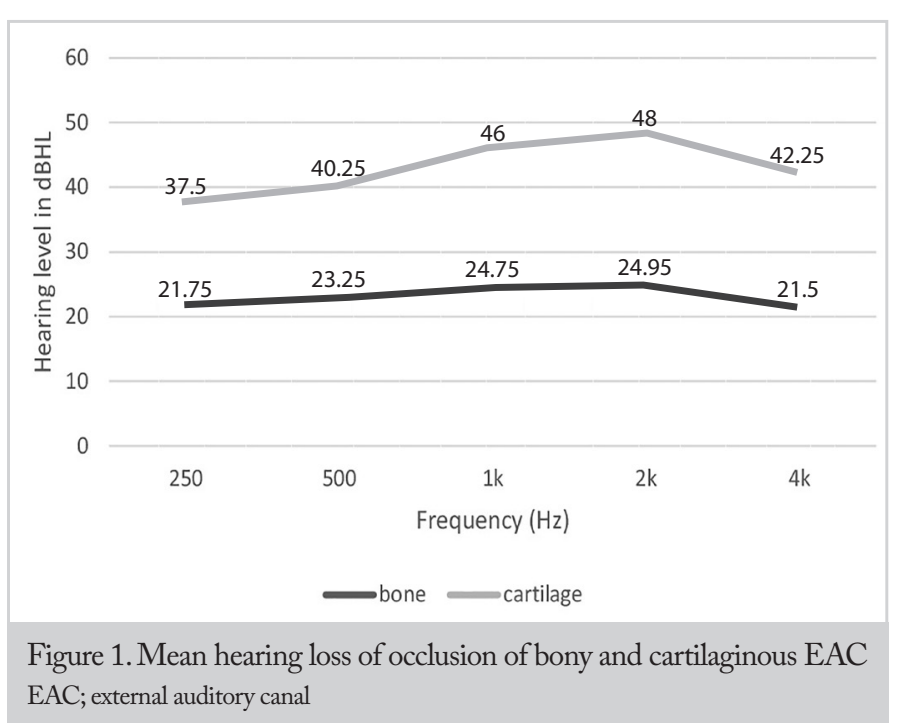


Table 3. Differences in the degree of hearing loss upon occlusion of the bony (B) and the cartilaginous (C) portion of the external canal at different frequencies

\begin{tabular}{|c|c|c|c|c|c|c|c|}
\hline \multirow[b]{2}{*}{ Pairwise comparison } & \multirow[b]{2}{*}{ Mean difference } & \multirow[b]{2}{*}{ SD } & \multirow[b]{2}{*}{$\mathrm{SE}$} & \multicolumn{2}{|c|}{$95 \% \mathrm{CI}$} & \multirow[b]{2}{*}{$\mathrm{T}$} & \multirow[b]{2}{*}{$\mathrm{p}$} \\
\hline & & & & Lower & Upper & & \\
\hline $\mathrm{C} 0.25-\mathrm{B} 0.25$ & 15.75 & 8.315 & 1.86 & 11.86 & 19.64 & 8.47 & $<0.001$ \\
\hline $\mathrm{C} 0.5-\mathrm{B} 0.5$ & 17.00 & 4.413 & 0.99 & 14.94 & 19.07 & 17.23 & $<0.001$ \\
\hline $\mathrm{C} 1-\mathrm{C} 1$ & 21.25 & 8.867 & 1.98 & 17.10 & 25.40 & 10.72 & $<0.001$ \\
\hline $\mathrm{C} 2-\mathrm{B} 2$ & 23.05 & 9.854 & 2.20 & 18.44 & 27.66 & 10.46 & $<0.001$ \\
\hline $\mathrm{C} 4-\mathrm{B} 4$ & 20.75 & 11.15 & 2.49 & 15.53 & 25.97 & 8.32 & $<0.001$ \\
\hline
\end{tabular}

SD: standard deviation; SE: standard error; CI: confidence interval

for the occlusion of bony EAC, there is a mild hearing loss at all frequencies as compared to cartilaginous EAC occlusion which reveals a mild to moderate hearing loss. The highest difference average was seen at $2000 \mathrm{~Hz}$ which was 23.05 , and the least difference at $250 \mathrm{~Hz}$ which was 15.75. The mean differences at 500, 1000 and 4000 were $17 \mathrm{~dB}, 21.25 \mathrm{~dB}$ and $20.75 \mathrm{~dB}$, respectively.

Table 3 shows the differences in the degree of hearing loss when compared to occlusion at the bony and the cartilaginous external auditory canals at different frequencies. Statistical analysis revealed that the occlusion data for both the bony and the cartilaginous EAC were normally distributed. The lowest frequency which was $0.25 \mathrm{kHz}$ showed a mean difference of 15.75 with a standard deviation of 8.315. The standardized difference was $\mathrm{t}=8$, and the two-tailed $\mathrm{p}$-value of the test was less than 0.001. The frequencies in between, i.e., $0.5 \mathrm{kHz}, 1 \mathrm{kHz}$ and 2 $\mathrm{kHz}$ showed a mean difference of $17,21.25$ and 23.05 with a standard deviation of 4.413, 8.867 and 9.854 , respectively. The two-tailed p-values were less than 0.05 . The highest frequency tested, which was $4 \mathrm{kHz}$, also revealed similar results. Thus, this concludes that there was a significant difference in the degree of hearing loss upon occlusion at the bony and the cartilaginous external auditory canals.

\section{Discussion}

There are several studies that were conducted in relation to the occlusion of the ear canal and the degree of hearing loss. Chandler (4) conducted the earliest study documenting ear canal occlusion and subsequent hearing loss. He substituted impression material for cerumen and tried to duplicate the occlusion caused by impacted cerumen. His subjects consisted of two patients only and he showed that when the ear canal is occluded from $80-100 \%$, threshold sensitivity at $2 \mathrm{kHz}$ and above is systematically reduced by an average of $13-20 \mathrm{~dB}(4)$.

Roeser and Ballachanda (5) did a study with five individuals in which they ascertained the degree of hearing loss using a combination of impression material and commercial lubricant. The authors grouped occlusion as complete occlusion, $60-80 \%$ occlusion and 40-60\% occlusion. In general, mean threshold differences across frequencies varied by as much as $55 \mathrm{~dB}$ to as little as $3 \mathrm{~dB}$. The most significant impact of occlusion was on frequencies from $2 \mathrm{kHz}$ through $8 \mathrm{kHz}$ regardless of the individual's condition (5).
Subha and Raman (6) conducted another study at the same institute in which 80 patients of different ethnicities with impacted cerumen underwent a PTA and the level of hearing loss was assessed. They computed a mean hearing loss of $21.1 \mathrm{~dB}$ due to impacted cerumen.

This study correlates the degree of hearing loss upon occluding the cartilaginous and the bony ear canals separately in one setting. The study that we have done demonstrated that the mean threshold of hearing loss upon occlusion of the cartilaginous portion is 37 to $48 \mathrm{~dB}$ with the highest loss at $2 \mathrm{kHz}$. The mean threshold of hearing loss of occlusion of the bony portion was $21.55 \mathrm{~dB}$ to $24.95 \mathrm{~dB}$. These findings may correlate to the concept and the properties of sound waves, resonance and impedance.

Sound waves need to travel through a medium such as solid, liquid or air. Sound waves move through each medium by vibrating the molecules in the matter. The molecules in solids are packed very tightly, and as for air, they are very loosely packed. The spacing of the molecules enables sound to travel much faster through solid than through air. Nonetheless, when sound travels through different mediums, there is a reflection as well as impedance. Impedance is the amount of opposition that a medium presents to sound waves trying to pass through and is affected by the compressibility and the density of the medium. A simplified equation is $Z=\mathrm{dxc}$ ( $Z$-acoustic impedance, d-density, $\mathrm{kg}$ / $\mathrm{m}^{3}$, c-speed of the wave, $\mathrm{m} / \mathrm{s}$ ) (7). Acoustic impedance is the physical property which describes the resistance of sound when it passes through a specific medium or tissue. Table 4 shows the acoustic impedance of various common mediums on ultrasound examination (8). The table demonstrates that air has the lowest impedance and bone the highest.

According to the above-given formula, the higher the density of the material, the higher the acoustic impedance. The principles and the formula can be applied in this study. The ear canal was occluded at two areas. One was at the cartilaginous portion of the external ear canal as depicted in Figure 2. Sound waves must travel through two different interfaces before reaching the tympanic membrane semi-solid material, represented by the aqueous cream, and air and these are two different mediums with two distinct acoustic impedance values. Thus, using the formula above, the cumulative acoustic impedance values result in a greater loss of sound energy, and this corresponds to the findings from this study. 
Table 4. Standard acoustic values of materials' medium impedance values (in standard unit)

\begin{tabular}{l|c}
\hline Medium & Impedance (in standard unit) \\
\hline Aira & 0.000429 \\
\hline Water & 1.50 \\
\hline Blood & 1.59 \\
\hline Fat & 1.38 \\
\hline Muscle & 1.70 \\
\hline Bone & 6.50 \\
\hline
\end{tabular}

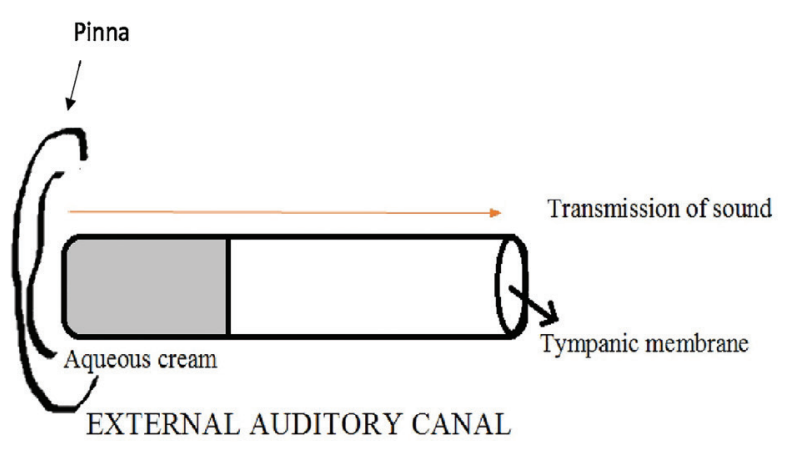

Figure 2. Cartilaginous canal block

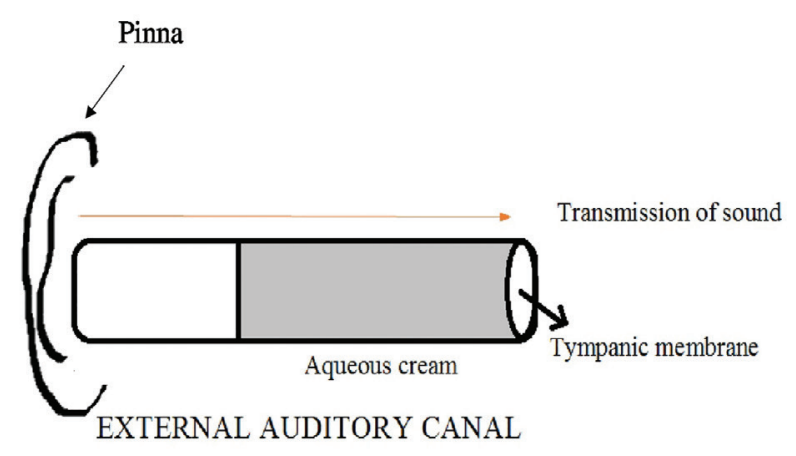

Figure 3. Bony ear canal block

As opposed to the cartilaginous canal block, depicted in Figure 3 , transmission of sound is from semi-solid component (aqueous cream) and then directly to the tympanic membrane, accounting for less degree of hearing loss.

As mentioned, in this study, the mean threshold of hearing loss upon occlusion of the cartilaginous portion was 37 to $48 \mathrm{~dB}$ with the highest loss at $2 \mathrm{kHz}$. The external auditory canal behaves as a resonator with the resonance frequency represented at a frequency range of $27 \mathrm{kHz}$, with an amplitude between 10 and $20 \mathrm{~dB}$ (9), and these frequencies are essential for speech recognition (10). This may correspond to the highest mean degree of hearing loss that was seen at $2 \mathrm{kHz}$.

The mean length of the ear canal is $27.7 \mathrm{~mm}$ with a medial twothirds bony portion and a lateral one-third cartilaginous portion
(11). The isthmus is an anatomical landmark corresponding to the bony cartilaginous junction. Acoustic Impedance depends on speed and density. The speed of sound is faster in solid materials and slowest in gases. However, sound passes through both air and semi-solid interface in either case, i.e., the occlusion of the cartilaginous or the bony EAC. Therefore, there is no definite relationship between the length of the occlusion and the degree of the hearing loss. Similarly, Subha and Raman (6) demonstrated a lack of association between the length of cerumen and the degree of hearing loss.

This study was conducted as an additional basic science knowledge of the application of physics of sounds in relation to the ear canal. The implications from this study may be related to conditions like keratosis obturans and canal cholesteatoma with blocking of the bony canal causing a milder hearing loss compared to occlusion of the cartilaginous portion with ear wax.

The limitation of this study is the unknown density of the aqueous cream, hence the impedance caused by this substance could not be assessed. In addition, an adequate seal was attained between the tympanic membrane and the isthmus via clinical judgement by examination under microscope. There is no tool that can be used to confirm a tight seal between the tympanic membrane and the isthmus.

\section{Conclusion}

Many studies have highlighted the degree of sound lost upon of the occlusion of the cartilaginous external ear canal. In this pilot study, we have highlighted the degree of hearing loss when both portions of the external ear canal are occluded. It is possible to conclude that a cartilaginous block of the external ear canal causes more hearing loss than a block of the bony ear canal.

Ethics Committee Approval: Ethics committee approval was received for this study from the Ethics Committee of Clinical Research of University Hospital (Date: 04.01.2017, No: 2016103-4317).

Informed Consent: Written informed consent was obtained from patients who participated in this study.

Peer-review: Externally peer-reviewed.

Author Contributions: Concept - R.R.; Design - R.R., D.A.N., A.W.C.; Supervision - R.R.; Resource - R.R., D.A.N.; Materials - R.R., D.A.N.; Data Collection and/or Processing - R.R., D.A.N., A.W.C.; Analysis and/or Interpretation - R.R., D.A.N.; Literature Search - R.R., D.A.N.; Writing - R.R., D.A.N., A.W.C.; Critical Reviews - R.R., A.C.W., D.A.N.

Conflict of Interest: The authors have no conflicts of interest to declare.

Financial Disclosure: The authors declared that this study has received no financial support.

\section{References}

1. Rossing TD, Moore FR, Wheeler PA. The Science of Sound 2002. 3rd edition. San Francisco: Addison Wesley. 
2. Ahmad I, Lee WC, Binnington JD. External auditory canal measurements: Localization of the isthmus. Otorhinolaryn Nova 2000; 10: 183-186. [CrossRef]

3. Casali, JG, Mauney DW, Burks JA. Physical vs. Psychophysical measurement of hearing protector attenuation - a.k.a. MIRE vs. REAT. Sound and Vibration 1995; 29: 20-7.

4. Chandler JR. Partial occlusion of the external auditory meatus: Its effect upon air and bone conduction hearing acuity. Laryngoscope 1964; 74: 22-54. [CrossRef]

5. Roeser RJ, Ballachanda BB. Physiology, pathophysiology, and anthropology/epidemiology of human earcanal secretions. J Am Acad Audiol 1997; 8: 391-400.

6. Subha ST, Raman R. Role of impacted cerumen in hearing loss. Ear Nose Throat J 2006; 85: 650, 652-3. [CrossRef]
7. Lotfi H,Faiz B, Moudden A. Characterization acoustic impedance of mortar using ultrasonic technique. Journal of Civil Engineering Research 2013; 3: 46-51.

8. Singh JP, Sharma R. Review of acoustic impedance variations with utrasonic velocity of various materials. International Journal of Pharmacy 2015; 5: 5-9.

9. Chisolm TH, Willott JF, Lister JJ. The ageing auditory system: anatomic and physiologic changes and implications for rehabilitation. Int J Audio 2003; 42: 3-10. [CrossRef]

10. Couto CM, Carvalho RMM. The effect external and middle ears have in otoacoustic emissions. Rev Bras Otorrinolaringol 2009; 75:15-23. [CrossRef]

11. Ahmad I, Lee WC, Binnington JD. External auditory canal measurements: Localization of the isthmus. Otorhinolaryngol Nova 2000; 10: 183-6. [CrossRef] 